\title{
Analysis of Effect of Surface Roughness of Electrically Conducting Fibre on its Backscattered RCS
}

\author{
Kishan Lal Gadri, Verandra Kumar, Ajit Kumar Singh, Alok Basita, Prashant Vasistha*, and Ravindra Kumar \\ Defence Laboratory, DRDO, Jodhpur, 342011, INDIA \\ *Corresponding Author: pvasistha@yahoo.com
}

\begin{abstract}
Presently, chaffs are widely used as electronic counter measure in deceiving tracking radar systems, since they have low production cost and yet they are very effective in creating false radar signatures. These chaffs are designed to cover radar frequency range from 2-18 GHz. However, in future, tracking radars will operate in higher frequency range and will have improved algorithms to mitigate chaff noise of certain Radar Cross Section (RCS) threshold. There are various factors to improve backscattered RCS of the chaff cloud at higher frequencies. In this paper, the effect of surface roughness of electrically conducting fibre (which is used as chaff) on its backscattered RCS has been studied. It has been observed that with an increase in surface roughness of the fibre there is decrease in its backscattered RCS. This relationship between fibre surface roughness and its backscattered RCS were obtained by performing simulations in Ansys HFSS with dipole length of $100 \mathrm{~mm}$ and $50 \mathrm{~mm}$ and later validating the same with the measured results for $50 \mathrm{~mm}$. Also it has been observed that with increase in frequency range the effect of surface roughness is more pronounce than at the lower frequencies for a given surface roughness range.
\end{abstract}

Keywords: - Backscattered RCS, electrically conducting fibre, HFSS simulations, Surface roughness.

\section{Introduction}

Since early days of World War II, metallic strips are being used as electronic counter measure to create false radar return to confuse enemy tracking radar systems. These metallic strips are known as Chaffs. Earlier these chaffs were consisted of $300 \mathrm{~mm}$ long and $15 \mathrm{~mm}$ wide strips [1], but nowadays these are reduced to few $\mathrm{mm}$ long with thickness in microns. The strips are cut to various lengths and each resonates at a particular radar frequency, thus by dispensing dipoles of several cut lengths, chaff can be made effective over a wide band of frequencies. These dipoles are also effective at harmonics of radar frequency [1] [2].

RCS behavior of any chaff payload depends upon various factors like chaff cut lengths, number of dipoles, mutual coupling, screening effects, polarization of EM waves, chaff ejection method etc [3]. Thus, for an effective chaff payload design, effects of all these parameters on RCS response must be analyzed and investigated using available simulation tools (like HFSS-IE, etc). Apart from these parameters, surface roughness of the electrically conducting fiber also affects its backscattered RCS response.

There are many research [1] [2] [3] on chaff RCS behavior on its various physical and electrical parameter variations. The average bistatic scattering cross section of thin cylindrical dipoles as a function of frequency, bistatic angle and polarization have been studied in [4]. The average backscattering cross section of clouds of linear metallic dipoles of resonant length and random orientation is investigated in [5]. A plane electromagnetic wave scattering by a straight resistive wire of finite length in an isotropic homogeneous medium is reported in [6]. A similar study of an electromagnetic wave scattering from both a perfectly conducting and lossy straight wire was also reported in [7]. Theoretical prediction and measurement of the scattering from a thin wire with one dimensional random orientation is analyzed in [8]. An approximate representation of the current along a perfectly conducting straight thin wire is reported in [9]. Transient analysis of straight thin wire scatterer by a multiresolution time domain integral equation method is reported in [10]. Another study on scattering characteristics of chaff for radar is reported in [11]. A new technique base on Inverse Synthetic Aperture Radar (ISAR) for the characterization of chaff elements is presented in [12]. A lot of work has been done on study of EM wave scattering from a thin dipole (a straight conducting wire) but there is no literature in which effect of chaff fibre surface roughness on its backscattered RCS is evaluated. In the present paper, relation between monostatic RCS of a chaff fiber with its surface roughness has been analyzed using simulations and latter validated with the measured results.

Radar cross section is a ratio of backscattered power per steradian in the direction of the radar from the target to the power density incident on the target. Mathematically, RCS can be represented by [13] [14]

$$
\sigma=\lim _{R \rightarrow \infty} 4 \pi R^{2}\left|\frac{s_{r}}{s_{t}}\right|
$$

Here, $R$ is the range, $S_{r}$ is the reflected power per steradian and $S_{t}$ is the transmitted power density.

Surface roughness (SR) is a feature of surface texture. It is quantified by the deviations in the direction of the normal vector of a surface from its ideal form. If these deviations are large, the surface is rough and if they are small, the surface is considered to be a smooth surface. There are various models available to quantify surface roughness like [15] or [16]. Recently, Huray et al. proposed a physics-based "snowball" model for surface roughness [17] [18]. Their model is based upon analytic solutions for the fields surrounding a hemispherical ball attached to a metal surface. The Huray model has been found to be more accurate than earlier approaches when the surface is very rough. In the 
present paper, simulations are performed in Ansys HFSS which is having two surface roughness models viz. Groisse model and Huray model. In this paper, Groisse model [16], which is considering surface roughness as a single value, has been used to model surface roughness of electrically conducting fibres. Higher the value of Groisse surface roughness parameter higher will be the surface roughness of the electrically conducting fibre.

Surface roughness of cylindrical metallic fibre can be also quantified by simply using the standard deviation of the diameter readings of the fibre being measured at multiple locations across the fibre axis. Higher the standard deviation of electrically conducting fibre diameter reading higher will be its surface roughness.

In this paper, effect of surface roughness of electrically conducting fibre on its backscattered RCS has been studied by performing RCS simulation in Ansys HFSS which is followed by RCS measurement of chaff dipole array with varying surface roughness in anechoic chamber using a time domain RCS measurement facility which gives RCS from 2 to $18 \mathrm{GHz}$ in single measurement. Here, in the study, comparison and analysis of simulated and measured RCS data is done at resonant frequency i.e. $3 \mathrm{GHz}$ for $50 \mathrm{~mm}$ chaff rather than the entire frequency range, since RCS at non-resonant frequencies is negligible as compared to RCS at resonant frequency. A good agreement in the simulations and measurements has been observed which validates the trend that with an increase in surface roughness of the fibre there is a decrease in its backscattered RCS. This relationship will help in optimizing tradeoffs between various chaff payload design parameters to improve its backscattered RCS response.

\section{Experimental setups and measurements}

\subsection{Fiber diameter measurement}

Direct measurements of electrically conducting fiber diameters are performed using Optical Microscope with resolution of 0.37 micron at $100 \mathrm{x}$ magnification [19]. Images of two individual fibers are shown in Fig. 1 and Fig. 2. The main advantage of using this method is capturing of actual variation of chaff dipole diameters. Using this method, 50 measurement readings were taken for each sample and then mean and standard deviation of the measured reading for each fiber are calculated. The mean and standard deviation of two fibers with large contrast in surface roughness (SR) are shown in Figure 1 Fig. 1and Figure 2. The mean and standard deviation of the fiber shown in Figure 1 are $24.59 \mu \mathrm{m}$ and $0.27 \mu \mathrm{m}$ respectively and for the fibre shown in Fig. 2 are $24.90 \mu \mathrm{m}$ and $2.42 \mu \mathrm{m}$ respectively. Table 1 lists mean and standard deviation of optical microscope measurements of diameters of the conducting fiber samples whose corresponding backscattered RCS measurements are carried out in anechoic chamber.

\subsection{Preparation of dipole array}

For a study of Backscattered RCS behavior of different chaff dipoles for a given sample, dipole arrays of fixed

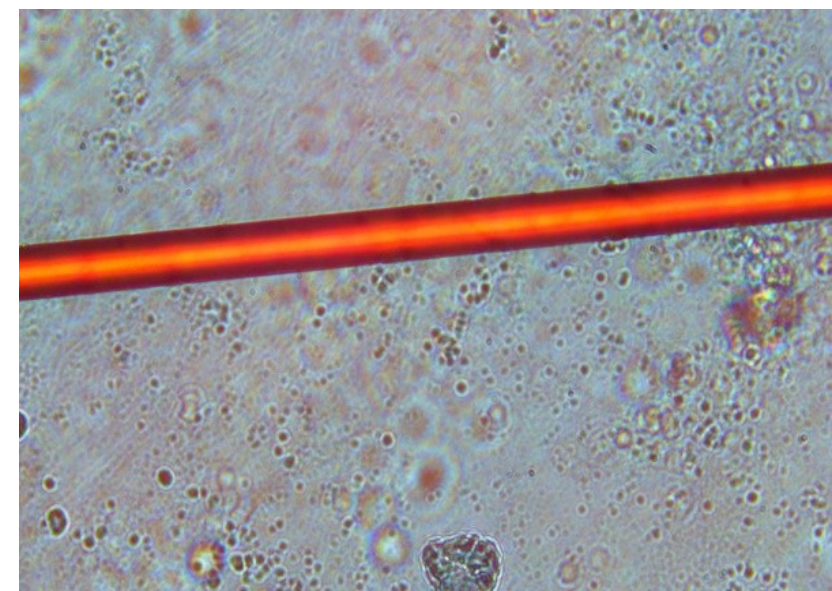

Fig. 1. Electrically conducting fibre with mean diameter of $24.59 \mu \mathrm{m}$ with standard deviation of $0.27 \mu \mathrm{m}$ (lower standard deviation).

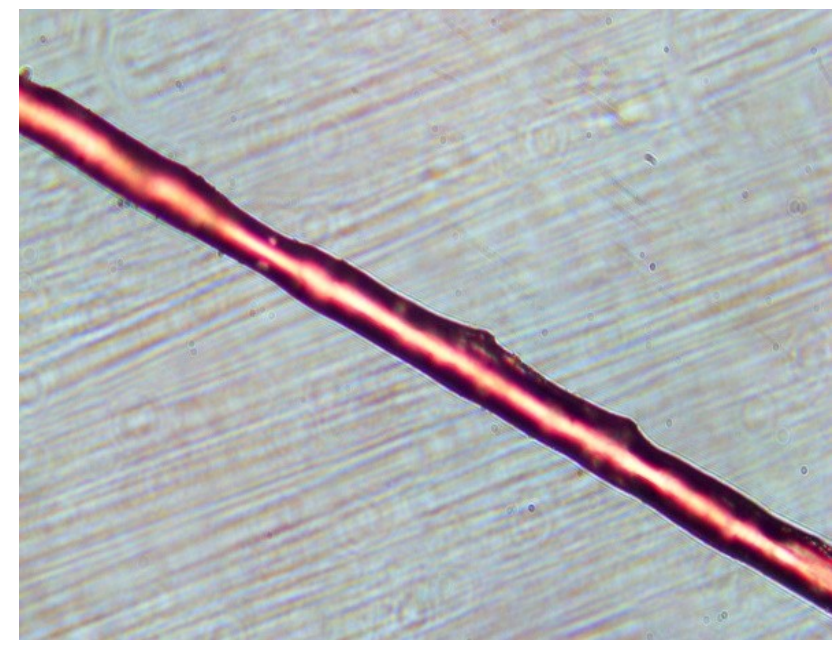

Fig. 2. Electrically conducting fibre with mean diameter of $24.90 \mu \mathrm{m}$ with standard deviation of $2.42 \mu \mathrm{m}$ (higher standard deviation).

Table 1: Mean and standard deviation of optical microscope measurements of conducting fibers used for res and surface roughness analysis.

\begin{tabular}{|l|l|l|l|}
\hline $\begin{array}{l}\text { Sample } \\
\text { No. }\end{array}$ & $\begin{array}{l}\text { Number of } \\
\text { Fibres in } \\
\text { each Dipole } \\
\text { Array }\end{array}$ & $\begin{array}{l}\text { Mean } \\
\text { Fiber } \\
\text { Diameter } \\
(\mu \mathrm{m})\end{array}$ & $\begin{array}{l}\text { Standard } \\
\text { Deviation of Fibre } \\
\text { Diameter } \\
\text { Readings }(\mu \mathrm{m})\end{array}$ \\
\hline 1 & 36 & 12.66 & 2.78 \\
\hline 2 & 36 & 11.68 & 1.29 \\
\hline 3 & 36 & 8.86 & 1.85 \\
\hline 4 & 36 & 9.61 & 0.95 \\
\hline 5 & 36 & 9.96 & 1.02 \\
\hline 6 & 36 & 11.10 & 0.99 \\
\hline 7 & 36 & 12.94 & 1.27 \\
\hline 8 & 36 & 9.68 & 0.90 \\
\hline 9 & 36 & 11.39 & 1.22 \\
\hline 10 & 36 & 10.39 & 1.57 \\
\hline 11 & 36 & 12.46 & 0.64 \\
\hline 12 & 36 & 14.34 & 1.11 \\
\hline 13 & 36 & 13.65 & 0.72 \\
\hline 14 & 36 & 14.38 & 0.78 \\
\hline 15 & 36 & 16.56 & 1.37 \\
\hline 16 & 36 & 16.89 & 1.37 \\
\hline 17 & 36 & 13.99 & 1.26 \\
\hline 18 & 36 & 25.17 & 0.46 \\
\hline & & & \\
\hline & & &
\end{tabular}


arrangements (horizontal and vertical spacing) of predefined dipole cut length $(50 \mathrm{~mm})$ are prepared on a microwave transparent thermocol sheet to evaluate effects of different surface roughness over its backscattered RCS. Figure 3 shows the schematic of prepared dipole array used for the experiments. Cut length of the chaff dipoles used is $50 \mathrm{~mm}$. The horizontal spacing between each dipole is $50 \mathrm{~mm}$ and the vertical spacing is $25 \mathrm{~mm}$.

\section{Results and observations}

\subsection{Simulated data}

Simulations are performed in Ansys HFSS [20] [21] using Integral Equation Method. Two different length of chaff dipole viz $100 \mathrm{~mm}$ and $50 \mathrm{~mm}$ are considered with varying Groisse surface roughness (SR) parameter from 0 to $1 \mu \mathrm{m}$. The simulation results for $100 \mathrm{~mm}$ and $50 \mathrm{~mm}$ chaff dipoles are shown in Figure 4 and 5, respectively. It can be easily observed that in both cases with increase in SR values there is a decrease in backscattered RCS of the electrically conducting fibre. Thus, indicating an inverse relation between surface roughness of the electrically conducting fiber and its backscattered RCS response.

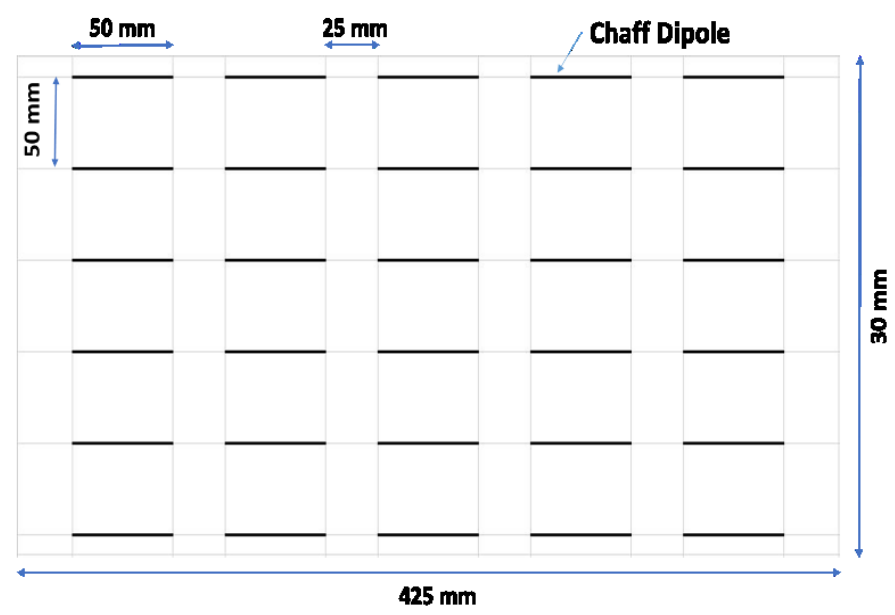

r1g. 3. scnematic or cnaII aipoie array arrangement $10 \times 0=50 \mathrm{cnaII}$ dipoles)

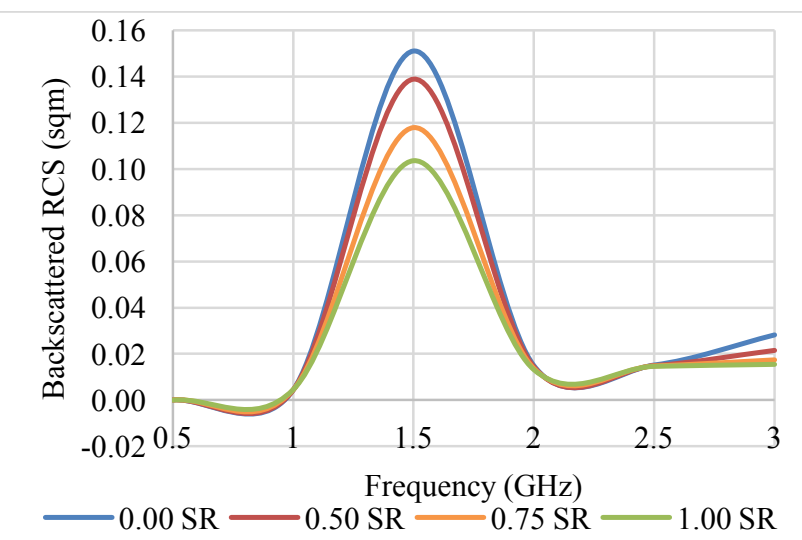

Fig. 4. Simulated backscattered RCS v/s fibre surface roughness (SR) for $100 \mathrm{~mm}$ electrically conducting fibre. unit of surface roughness (SR) parameter is in micron $(\mu \mathrm{m})$.

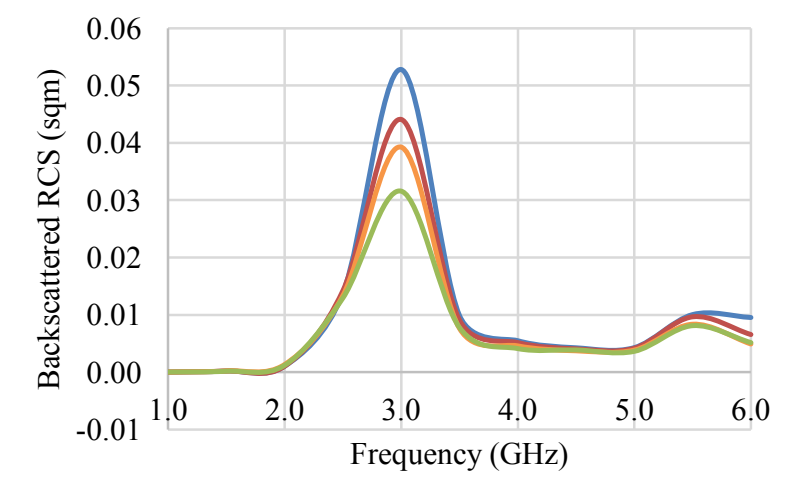

$-0.00 \mathrm{SR}-0.50 \mathrm{SR}-0.75 \mathrm{SR}-1.00 \mathrm{SR}$

Fig. 5. Simulated backscattered RCS v/s fibre surface roughness (SR) for $50 \mathrm{~mm}$ electrically conducting fibre. unit of surface roughness (SR) parameter is in micron $(\mu \mathrm{m})$.

\subsection{Measured data}

In the present study, time domain techniques are used for backscattered RCS measurements. In Time domain (TD) technique, ultra-wide band pulse of $\sim 30$ picosecond is used to carry out measurements which gives monostatic RCS over a wide frequency range of $2-18 \mathrm{GHz}$ in single measurement. Figure 6 shows the experimental setup for TD static RCS measurements of chaff dipole array in anechoic chamber.

Since it is not feasible to perform RCS measurement of bloomed metallic fiber cloud in anechoic chamber, therefore a different approach for test coupon fabrication has been thought off. For this, metallic fibers are placed on microwave transparent 2D thermocol sheet (schematic is as shown in Figure 3) and measurements are performed. Measured data are shown in Table 2. There are three parameters of electrically conducting fibers which are measured and reported in Table 2 are namely, mean fibre diameter, its corresponding standard deviation of the fiber diameter readings and Backscattered RCS. It must be noted that all the data has been normalized with respect to the highest value in each column.

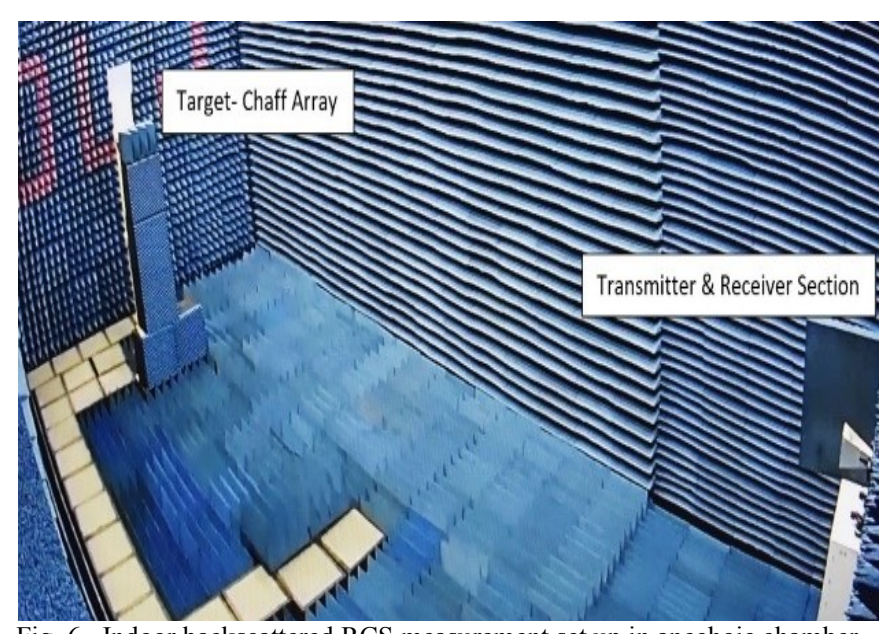

Fig. 6. Indoor backscattered RCS measurement set up in anechoic chamber 
Table 2: measured backscattered RCS and its corresponding electrically conducting fibre diameter and their standard deviation normalized with their corresponding highest values

\begin{tabular}{|l|l|l|}
\hline $\begin{array}{l}\text { Normalized } \\
\text { Mean Fibre } \\
\text { Diameter }\end{array}$ & $\begin{array}{l}\text { Normalized Standard } \\
\text { Deviation of Fibre } \\
\text { Diameter readings }\end{array}$ & $\begin{array}{l}\text { Normalized } \\
\text { Backscattered RCS }\end{array}$ \\
\hline 0.503 & 1.000 & 0.282 \\
\hline 0.464 & 0.462 & 0.499 \\
\hline 0.352 & 0.664 & 0.144 \\
\hline 0.382 & 0.343 & 0.173 \\
\hline 0.395 & 0.365 & 0.178 \\
\hline 0.441 & 0.356 & 0.485 \\
\hline 0.514 & 0.455 & 0.258 \\
\hline 0.384 & 0.324 & 0.203 \\
\hline 0.453 & 0.439 & 0.456 \\
\hline 0.413 & 0.562 & 0.415 \\
\hline 0.495 & 0.228 & 0.692 \\
\hline 0.570 & 0.399 & 0.688 \\
\hline 0.542 & 0.258 & 0.655 \\
\hline 0.571 & 0.279 & 0.646 \\
\hline 0.658 & 0.492 & 0.713 \\
\hline 0.671 & 0.493 & 0.790 \\
\hline 0.556 & 0.452 & 0.771 \\
\hline 1.000 & 0.166 & 1.000 \\
\hline
\end{tabular}

In order to estimate effect of fiber diameter and fiber surface roughness (represented by standard deviation of the fiber diameter readings) a multiple linear regression is performed on measured data of Table 2. Linear Regression Analysis of Measured Values (i.e., Normalized Mean Fiber Diameter $(\mu \mathrm{m})$, its Standard Deviation $(\mu \mathrm{m})$ and Normalized Backscattered RCS, - are performed in the form of following equation (1)-

$$
R C S=a * F i b \_D i a+b * S t d \_D e v+c
$$

Here- $\mathrm{a}, \mathrm{b} \& \mathrm{c}=$ linear regression coefficient;

Fib Dia=Normalized Mean Diameter of the fibre $(\mu \mathrm{m})$, Std_Dev $=$ Normalized Standard Deviation of the fibre diameter readings. Here, Std_Dev indicates the roughness of the Fibre \& RCS = Backscattered RCS of the Fibre Array.

The result of linear regression is given in Table 3. It can be observed that the coefficient for the mean fiber diameter ' $a$ ' is positive (1.31) indicating that with increase in fibre diameter there will be increase in backscattered RCS of the electrically conducting fibre while the coefficient for the fiber surface roughness i.e. ' $b$ ' is negative (-0.24) indicating that with increase in fibre roughness there will be a decrease in its corresponding backscattered RCS of electrically conducting fibre (also shown in Figure 7). So, in order to get maximum backscattered RCS from a given chaff fibre, the fiber must be as smooth as possible.

Table 3: Values of Coefficients after performing regression on normalized measured data

\begin{tabular}{|l|l|}
\hline Coefficient & Values \\
\hline a & 1.31 \\
\hline b & -0.24 \\
\hline c & -0.08 \\
\hline Adjusted R Square & 0.68 \\
\hline
\end{tabular}

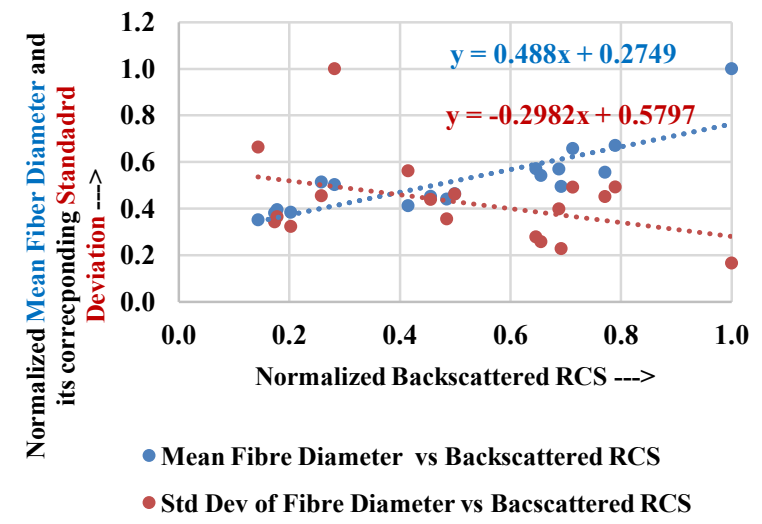

Figure 7 Relationship observed between normalized values of backscattered RCS vs electrically conducting fibre diameter and backscattered RCS vs fiber diameter standard deviation

It is observed from both simulated and measured data that with an increase in fiber surface roughness there is a decrease in its backscattered RCS. There may be two possible reasons for this trend- first with an increase in its surface roughness, specular backscattering reduces which results in lower values of backscattered RCS. Secondly, with increase in surface roughness there is an increase in the metal surface area relative to a smooth surface which will certainly affects current transport, increasing the observed loss. This is particularly true when the skin depth becomes comparable to or less than the average height of the surface roughness [22] [23]. A typical standard deviation of these diameter readings is in the range of 0.5 to 2 microns.

From the RCS simulation graphs of $100 \mathrm{~mm}$ and $50 \mathrm{~mm}$ fiber one can also estimate percentage reduction in backscattered RCS for a given range of surface roughness (SR) $(0-1 \mu \mathrm{m})$ with frequency. It can be observed form Figure 4 at $\mathrm{SR}=0 \mu \mathrm{m}$ the backscattered RCS value is 0.150 sqm and at maximum $\mathrm{SR}=1 \mu \mathrm{m}$ the backscattered RCS value is $0.101 \mathrm{sqm}$ at $1.5 \mathrm{GHz}$ resonant frequency. So, the percentage reduction in backscattered RCS at $1.5 \mathrm{GHz}$ is $[(0.150-0.101) / 0.150] * 100 \sim 32.67 \%$. Similarly, percentage reduction in backscattered RCS at $3 \mathrm{GHz}$ is $40 \%$. Thus, it can be clearly be observed that percentage reduction in backscattered RCS is more at higher frequencies than at lower frequencies for a given surface roughness (SR) range. The skin depth in any good conducting metal copper/aluminum at $1 \mathrm{GHz}$ is around 2 microns, so the surface roughness effects will become more prominent for multi-gigahertz backscattered RCS values as the skin depth becomes more comparable to the surface roughness.

\section{Conclusion}

In the present paper, effect of surface roughness of electrically conducting fibre on its corresponding backscattered RCS has been studied. It is observed from both the simulation and measured results that with an increase in roughness of the electrically conducting fibre surface there is a decrease in its corresponding backscattered RCS response. Thus, if other parameters of electrically conducting fibers are kept constant then its backscattered 
RCS can be improved with increasing smoothness of the electrically conducting fibers. It is also observed that with a given increase in surface roughness the percentage reduction in its corresponding backscattered RCS is more at higher frequencies. Thus the effects of the surface roughness will be more pronounced at higher frequencies.

\section{References}

[1] B. C. Butters, Chaff, IEE Procedings, vol. 129, no. 3, pp. 197201, June 1982.

[2] V. Kumar, A. K. Singh, P. Vasistha, and R. Kumar, Dynamic RCS prediction of rapidly blooming chaff cloud and its validation using measurement on scaled down, International Journal of Advances in Microwave Technology (IJAMT), vol. 3, no. 4, pp. 170-175, 2018.

[3] S. W. Marcus, Dynamics and radar cross section density of chaff clouds, IEEE Transaction on Aerospace and Electronic Systems, vol. 40, no. 1, pp. 93-102, 2004.

[4] C. L. Mack and B. Reiffen, RF characteristics of thin dipoles, Proceeding of IEEE, pp. 533-542, 1964.

[5] R. G. Wickliff and R. J. GarBacz, The average backscattering cross section of clouds of randomized resonant dipoles, IEEE Transactions on Antennas and Propagation, vol. 22, no.3, pp. 503-505, 1974.

[6] O. Einarsson and T. Plato, Electromagnetic scattering by a thin resistive wire, Electronic Letters, vol. 5, no. 25, pp. 10191025, Dec 1969.

[7] H. T. Shamansky, A. K. Dminek, and L. Peters, Electromagnetic scattering by a straight thin wire, IEEE Transactions on Antennas and Propagation, vol. 37, no. 8, pp. 1019-1025, 1989.

[8] P. Snoeij and P. J. Swart, Theoritical prediction and measurement of the scattering from thin wires with one dimensional random orientation, Proceedings of IGARSS '94, vol. 1, pp. 87-89, 1994.

[9] J. C. Bogerd, A. G. Tijhuis, and J. A. Klaasen, Electromagnetic excitation of a thin wire: a travelling wave approach, IEEE Transaction on Antennas and Propagation, vol. 46, no. 8, pp. 1202-1211, 1998.

[10] Z. Zhou and J. S. Tyo, Transient analysis of straight thin wire scatterer by multiresolution time domain integral equation method, IEEE Antenna and Propagation Society, vol. 3, pp. 575-578, 2003.

[11] Z. Yinan, J. Ming, Q. Xiaolin, and Z. Zhiquan, Study on scattering characteristics of chaff for radar, 4th International Conference on Microwave and Millimeter Wave Technology Proceedings, 2004.

[12] D. Scholfield, M. Myat, J. Dauby, J. Fesler, and J. Bright, A new technique for the characterization of chaff elements, The Review of Scientific Instruments, vol. 82, no. 7, pp. 074702, 2011.

[13] E. F. Knott, Radar Cross Section Fundamentals, Radar Cross Section Measurements, First ed., New York, SciTech, 1993, pp. 14-17.

[14] M. L. Skolnik, Radar Cross Section of Targets, in Introduction to RADAR Systems, Second ed., Tata McGraw Hill, 1981, pp. 33-45.

[15] E. Hammerstad and O. Jensen, Accurate models for microstrip computer-aided design, in IEEE MTT-S Intl. Microwave Symposium Digest, Washington, DC, 1980.

[16] S. Groiss, I. Bardi, O. Biro, K. Preis, and K. R. Richter, Parameters of lossy cavity resonators calculated by the finite element method, IEEE Transactions on Magnetics, vol. 32, no. 3, pp. 894-897, 1996.

[17] S. Hall, S. G. Pytel, P. G. Huray, D. Hua, A. Moonshiram, G. A. Brist, and E. Sijercic, Multigigahertz causal transmission line modeling methodology using a 3-D hemispherical surface roughness approach, IEEE Tranactions on Microwave Theory and Techniques, vol. 55, no. 12, pp. 2614-2624, 2007.

[18] P. G. Huray, O. Oluwafemi, J. Loyer, E. Bogatin, and X. Ye, Impact of copper surface texture on loss: a model that works, DesignCon 2010 Proceedings, Santa Clara, CA, 2010.
[19] Olympus, Performing Measurements, in User Manual Olympus Stream Image Processing Software, Olympus, pp. 48-51.

[20] Ansys Inc., Fundamentals of HFSS, in An Introduction to HFSS, Ansys Inc, 2020, pp. 1-12.

[21] Ansys Inc., Multi Frequency Adaptive Meshing, in An Introduction to Multi Frequency Adaptive Meshing in HFSS, Ansys, Inc., 2017, pp. 1-12.

[22] G. Gold and K. Helmreich, a physical surface roughness model and its applications, IEEE Transaction on Microwave Theory and Techniques, vol. 65, no. 10, pp. 3720-3732, October 2017.

[23] L. Tsang, X. Gu and H. Braunisch, Effect of random rough surface on absorption by conductors at microwave frequencies, IEEE Microwave and Wireless Components Letters vol. 16, no. 4, pp. 221-223, 2006.

\section{Biography of the authors}

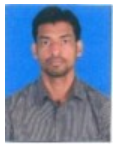

Kishan Lal Gadri: He did his B.Tech from Jaipur National University, Jaipur in ECE during 2007-2011. $\mathrm{He}$ has the experience of 08 years in the field of microwaves and its applications in remote sensing and modelling, simulation and RCS measurement of various electronic countermeasure systems at DRDO.

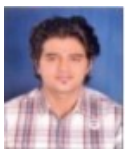

Verandra Kumar: He did his B Tech from MNIT, Jaipur in ECE during 2005-09. He joined DRDO in 2009 at DL, Jodhpur and currently working as Scientist D. His area of work includes RCS measurement and simulation of tagrets for stealth application, design of elecytronic countermeasure decoys as well as Dynamic RCS modelling, simulation and measurements of decoys currently being used by IAF and IN.

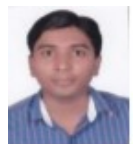

Ajit Kumar Singh: He joined DRDO in 2013 at DL, Jodhpur. He has the experience of 07 years in the area of design and development of software to simulate the parameters of electronic countermeasure and analysis of various chaff decoys used by IAF and IN.

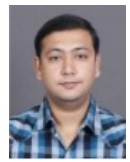

Alok Basita: He did his B.Tech from MNIT during 2001-2005. He joined DRDO in 2005 and currently working as scientist $\mathrm{E}$ at DL, Jodhpur. He has 15 years of experience in design of digital control systems for testing facility of various subsystems related to Gas Turbine Engine, electronic counter measure dispensing system, as well as RCS measurement studies on various targets and decoys suitable for electronic counter measure system.

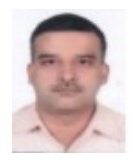

Prashant Vasistha: He did his PhD from Department of Electronics Engineering, IIT BHU. He joined DRDO in 1998 at DL, Jodhpur and currently working as Scientist G. He has been working in the area of radar cross section (RCS) management and charectrization studies and RCS measurement technologies for combat systems. He has an experience of 20 years in the area of design and development of radar camouflage techniques and electronic counter measure decoys.

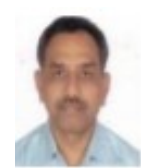

Ravindra Kumar: He did his BE from IIT Roorkee in 1983 and M Tech from IIT Mumbai in 1987. He joined DRDO in 1983 at DL, Jodhpur and currently working as Outsatnding scientist and Director of estalishment. He has an R\&D experience of 37 years in the area of desert warfare scenraio management, Phase change materials, water purifications systems and design, development and testing of electronic countermeasures. 